\title{
A NEW TYPE OF CONNECTED SETS VIA BIOPERATIONS
}

\author{
HARIWAN Z. IBRAHIM* \\ Department of Mathematics, Faculty of Education, University of Zakho, Kurdistan-Region, Iraq
}

*Corresponding author: hariwan_math@yahoo.com

\begin{abstract}
The purpose of this paper is to introduce the notion of $\alpha_{\left(\gamma, \gamma^{\prime}\right)}$-separated sets and study their properties in topological spaces, then we introduce the notions of $\alpha_{\left(\gamma, \gamma^{\prime}\right)}$-connected and $\alpha_{\left(\gamma, \gamma^{\prime}\right)}$-disconnected sets. We discuss the characterizations and properties of $\alpha_{\left(\gamma, \gamma^{\prime}\right)}$-connected sets and then properties under $\left(\alpha_{\left(\gamma, \gamma^{\prime}\right)}, \alpha_{\left(\beta, \beta^{\prime}\right)}\right)$-continuous functions. The $\alpha_{\left(\gamma, \gamma^{\prime}\right)}$-components in a space $X$ is also introduced.
\end{abstract}

\section{Introduction}

Njastad [5] introduced $\alpha$-open sets in a topological space and studied some of their properties. Ibrahim [1] introduced and discussed an operation of a topology $\alpha O(X)$ into the power set $P(X)$ of a space $X$ and also in [2] he introduced the notion of $\alpha O(X, \tau)_{\left(\gamma, \gamma^{\prime}\right)}$, which is the collection of all $\alpha_{\left(\gamma, \gamma^{\prime}\right)}$-open sets in a topological space $(X, \tau)$. In addition, Ibrahim [3] introduced the concept of $\left(\alpha_{\left(\gamma, \gamma^{\prime}\right)}, \alpha_{\left(\beta, \beta^{\prime}\right)}\right)$-closed and $\left(\alpha_{\left(\gamma, \gamma^{\prime}\right)}, \alpha_{\left(\beta, \beta^{\prime}\right)}\right)$-continuous functions and investigated some of their basic properties. Mishra [4] introduced $\alpha$ - $\tau$-disconnectedness and $\alpha$ - $\tau$-connectedness in topological spaces. In this paper, the author introduce and study the characterizations and properties of $\alpha_{\left(\gamma, \gamma^{\prime}\right)}$-connected and $\alpha_{\left(\gamma, \gamma^{\prime}\right)^{\prime}}$-disconnected spaces and then properties under $\left(\alpha_{\left(\gamma, \gamma^{\prime}\right)}, \alpha_{\left(\beta, \beta^{\prime}\right)}\right)$-continuous functions.

Received 2018-01-18; accepted 2018-03-19; published 2018-07-02.

2010 Mathematics Subject Classification. Primary 22A05, 22A10, Secondary 54C05.

Key words and phrases. $\alpha$-open; bioperations; $\alpha_{\left(\gamma, \gamma^{\prime}\right)}$-connected set; $\alpha_{\left(\gamma, \gamma^{\prime}\right)}$-disconnected set.

(C) 2018 Authors retain the copyrights of their papers, and all open access articles are distributed under the terms of the Creative Commons Attribution License. 


\section{Preliminaries}

Throughout the present paper, $(X, \tau)$ and $(Y, \sigma)$ (or simply $X$ and $Y$ ) denotes a topological spaces on which no separation axioms is assumed unless explicitly stated. For a subset $A$ of a space $X, C l(A)$ and $\operatorname{Int}(A)$ represent the closure of $A$ and the interior of $A$, respectively.

Definition 2.1. [5] A subset $A$ of a topological space $(X, \tau)$ is said to be $\alpha$-open if $A \subseteq \operatorname{Int}(C l(\operatorname{Int}(A)))$. The complement of an $\alpha$-open set is said to be $\alpha$-closed. The family of all $\alpha$-open (resp., $\alpha$-closed) sets in a topological space $(X, \tau)$ is denoted by $\alpha O(X, \tau)$ (resp., $\alpha C(X, \tau)$ ).

The intersection of all $\alpha$-closed sets containing $A$ is called the $\alpha$-closure of $A$ and is denoted by $\alpha C l(A)$.

Definition 2.2. [4] The subsets $A$ and $B$ of a topological space $(X, \tau)$ are called $\alpha$ - $\tau$-separated sets if $(\alpha C l(A) \cap B) \cup(A \cap \alpha C l(B))=\phi$.

Definition 2.3. [1] An operation $\gamma: \alpha O(X, \tau) \rightarrow P(X)$ is a mapping satisfying the condition, $V \subseteq V^{\gamma}$ for each $V \in \alpha O(X, \tau)$. We call the mapping $\gamma$ an operation on $\alpha O(X, \tau)$. The operation id: $\alpha O(X, \tau) \rightarrow P(X)$ is defined by $V^{i d}=V$ for any set $V \in \alpha O(X, \tau)$. This operation is called the identity operation on $\alpha O(X, \tau)$.

Definition 2.4. [2] A nonempty subset $A$ of $(X, \tau)$ is said to be $\alpha_{\left(\gamma, \gamma^{\prime}\right)}$-open if for each $x \in A$, there exist $\alpha$-open sets $U$ and $V$ of $X$ containing $x$ such that $U^{\gamma} \cup V^{\gamma^{\prime}} \subseteq A$. A subset $F$ of $(X, \tau)$ is said to be $\alpha_{\left(\gamma, \gamma^{\prime}\right)}$-closed if its complement $X \backslash F$ is $\alpha_{\left(\gamma, \gamma^{\prime}\right)}$-open. The set of all $\alpha_{\left(\gamma, \gamma^{\prime}\right)}$-open sets of $(X, \tau)$ is denoted by $\alpha O(X, \tau)_{\left(\gamma, \gamma^{\prime}\right)}$.

Definition 2.5. [2] Let $A$ be a subset of a topological space $(X, \tau)$.

(1) The union of all $\alpha_{\left(\gamma, \gamma^{\prime}\right)}$-open sets contained in $A$ is called the $\alpha_{\left(\gamma, \gamma^{\prime}\right)}$-interior of $A$ and is denoted by $\alpha_{\left(\gamma, \gamma^{\prime}\right)} \operatorname{Int}(A)$

(2) The intersection of all $\alpha_{\left(\gamma, \gamma^{\prime}\right)}$-closed sets containing $A$ is called the $\alpha_{\left(\gamma, \gamma^{\prime}\right)}$-closure of $A$ and denoted by $\alpha_{\left(\gamma, \gamma^{\prime}\right)}-C l(A)$.

Proposition 2.1. [2] Let $A$ and $B$ be subsets of $(X, \tau)$. Then the following hold:

(1) $A \subseteq \alpha_{\left(\gamma, \gamma^{\prime}\right)}-C l(A)$.

(2) If $A \subseteq B$, then $\alpha_{\left(\gamma, \gamma^{\prime}\right)}-C l(A) \subseteq \alpha_{\left(\gamma, \gamma^{\prime}\right)}-C l(B)$.

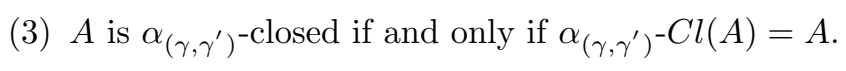

(4) $\alpha_{\left(\gamma, \gamma^{\prime}\right)}-C l(A)$ is $\alpha_{\left(\gamma, \gamma^{\prime}\right)}$-closed.

Proposition 2.2. [2] For a point $x \in X, x \in \alpha_{\left(\gamma, \gamma^{\prime}\right)}-C l(A)$ if and only if $V \cap A \neq \phi$ for every $\alpha_{\left(\gamma, \gamma^{\prime}\right)}$-open set $V$ containing $x$.

Definition 2.6. [3] A function $f:(X, \tau) \rightarrow(Y, \sigma)$ is said to be $\left(\alpha_{\left(\gamma, \gamma^{\prime}\right)}, \alpha_{\left(\beta, \beta^{\prime}\right)}\right)$-closed if for $\alpha_{\left(\gamma, \gamma^{\prime}\right)}$-closed set $A$ of $X, f(A)$ is $\alpha_{\left(\beta, \beta^{\prime}\right)}$-closed in $Y$. 
Proposition 2.3. [3] Let $f:(X, \tau) \rightarrow(Y, \sigma)$ be a function. Then, $f$ is $\left(\alpha_{\left(\gamma, \gamma^{\prime}\right)}, \alpha_{\left(\beta, \beta^{\prime}\right)}\right)$-closed if and only if $\alpha_{\left(\beta, \beta^{\prime}\right)}-C l(f(A)) \subseteq f\left(\alpha_{\left(\gamma, \gamma^{\prime}\right)^{-}} C l(A)\right)$ for every subset $A$ of $X$.

Theorem 2.1. [3] Suppose that $f:(X, \tau) \rightarrow(Y, \sigma)$ is $\left(\alpha_{\left(\gamma, \gamma^{\prime}\right)}, \alpha_{\left(\beta, \beta^{\prime}\right)}\right)$-continuous. Then,

(1) $f^{-1}(V)$ is $\alpha_{\left(\gamma, \gamma^{\prime}\right)}$-open for every $\alpha_{\left(\beta, \beta^{\prime}\right)}$-open set $V$ of $(Y, \sigma)$.

(2) For each point $x \in X$ and each $\alpha_{\left(\beta, \beta^{\prime}\right)}$-open $W$ of $(Y, \sigma)$ containing $f(x)$, there exist $\alpha_{\left(\gamma, \gamma^{\prime}\right)}$-open $U$ of $(X, \tau)$ containing $x$ such that $f(U) \subseteq W$.

\section{3. $\alpha_{\left(\gamma, \gamma^{\prime}\right)}$-Connected And $\alpha_{\left(\gamma, \gamma^{\prime}\right)}$-Disconnected Sets}

Throughout this section, let $\gamma, \gamma^{\prime}: \alpha O(X, \tau) \rightarrow P(X)$ be operations on $\alpha O(X, \tau)$ and $\beta, \beta^{\prime}: \alpha O(Y, \sigma) \rightarrow$ $P(Y)$ be operations on $\alpha O(Y, \sigma)$.

Definition 3.1. Two subsets $A$ and $B$ of a topological space $(X, \tau)$ are called $\alpha_{\left(\gamma, \gamma^{\prime}\right)^{-}}$separated if $\left(\alpha_{\left(\gamma, \gamma^{\prime}\right)^{-}}\right.$ $C l(A) \cap B) \cup\left(A \cap \alpha_{\left(\gamma, \gamma^{\prime}\right)^{-}} C l(B)\right)=\phi$.

Remark 3.1. Each two $\alpha_{\left(\gamma, \gamma^{\prime}\right)}$-separated sets are always disjoint, since $A \cap B \subseteq A \cap \alpha_{\left(\gamma, \gamma^{\prime}\right)}-C l(B)=\phi$. The converse may not be true in general, as it is shown in the following example.

Example 3.1. Let $X=\{1,2,3\}$ and $\tau=\{\phi, X,\{2\}\}$. For each $A \in \alpha O(X)$, we define two operations $\gamma$ and $\gamma^{\prime}$, respectively, by

$$
A^{\gamma}=A^{\gamma^{\prime}}= \begin{cases}A & \text { if } 3 \in A \\ X & \text { if } 3 \notin A .\end{cases}
$$

Since $\alpha O(X, \tau)_{\left(\gamma, \gamma^{\prime}\right)}=\{\phi, X,\{2,3\}\}$, then $\{2\}$ and $\{3\}$ are disjoint subsets of $X$, but not $\alpha_{\left(\gamma, \gamma^{\prime}\right)}$-separated.

From the fact that $\alpha C l(A) \subseteq \alpha_{\left(\gamma, \gamma^{\prime}\right)}-C l(A)$, for every subset $A$ of $X$. Then every $\alpha_{\left(\gamma, \gamma^{\prime}\right)}$-separated set is $\alpha$ - $\tau$-separated. But the converse may not be true as shown in the following example.

Example 3.2. Let $X=\{1,2,3,4\}$ and $\tau=\{\phi, X,\{1\},\{2\},\{1,2\}\}$. For each $A \in \alpha O(X)$, we define two operations $\gamma$ and $\gamma^{\prime}$, respectively, by

$$
A^{\gamma}=A^{\gamma^{\prime}}= \begin{cases}A & \text { if } 4 \in A \\ X & \text { if } 4 \notin A .\end{cases}
$$

Since $\alpha O(X, \tau)_{\left(\gamma, \gamma^{\prime}\right)}=\{\phi, X,\{1,2,4\}\}$, then the subsets $\{3\}$ and $\{4\}$ are $\alpha$ - $\tau$-separated, but not $\alpha_{\left(\gamma, \gamma^{\prime}\right)^{-}}$ separated.

Theorem 3.1. If $A$ and $B$ are any two nonempty subsets in a space $X$, then the following statements are true:

(1) If $A$ and $B$ are $\alpha_{\left(\gamma, \gamma^{\prime}\right)}$-separated, $A_{1} \subseteq A$ and $B_{1} \subseteq B$, then $A_{1}$ and $B_{1}$ are also $\alpha_{\left(\gamma, \gamma^{\prime}\right)}$-separated. 
(2) If $A \cap B=\phi$ such that each of $A$ and $B$ are both $\alpha_{\left(\gamma, \gamma^{\prime}\right)}$-closed $\left(\alpha_{\left(\gamma, \gamma^{\prime}\right)}\right.$-open), then $A$ and $B$ are $\alpha_{\left(\gamma, \gamma^{\prime}\right)}$-separated.

(3) If each of $A$ and $B$ is $\alpha_{\left(\gamma, \gamma^{\prime}\right)}$-closed $\left(\alpha_{\left(\gamma, \gamma^{\prime}\right)}\right.$-open) and if $H=A \cap(X \backslash B)$ and $G=B \cap(X \backslash A)$, then $H$ and $G$ are $\alpha_{\left(\gamma, \gamma^{\prime}\right)}$-separated.

Proof. (1) Since $A_{1} \subseteq A$, then $\alpha_{\left(\gamma, \gamma^{\prime}\right)^{-}} C l\left(A_{1}\right) \subseteq \alpha_{\left(\gamma, \gamma^{\prime}\right)^{-}} C l(A)$. Then, $B \cap \alpha_{\left(\gamma, \gamma^{\prime}\right)^{-}} C l(A)=\phi$ implies $B_{1} \cap \alpha_{\left(\gamma, \gamma^{\prime}\right)^{-}} C l(A)=\phi$ and $B_{1} \cap \alpha_{\left(\gamma, \gamma^{\prime}\right)^{-}} C l\left(A_{1}\right)=\phi$. Similarly $A_{1} \cap \alpha_{\left(\gamma, \gamma^{\prime}\right)}-C l\left(B_{1}\right)=\phi$. Hence, $A_{1}$ and $B_{1}$ are $\alpha_{\left(\gamma, \gamma^{\prime}\right)}$-separated.

(2) Since $A=\alpha_{\left(\gamma, \gamma^{\prime}\right)}-C l(A), B=\alpha_{\left(\gamma, \gamma^{\prime}\right)^{-}} C l(B)$ and $A \cap B=\phi$, then $\alpha_{\left(\gamma, \gamma^{\prime}\right)^{-}} C l(A) \cap B=\phi$ and $\alpha_{\left(\gamma, \gamma^{\prime}\right)} C l(B) \cap A=\phi$. Hence, $A$ and $B$ are $\alpha_{\left(\gamma, \gamma^{\prime}\right)}$-separated. If $A$ and $B$ are $\alpha_{\left(\gamma, \gamma^{\prime}\right)}$-open, then

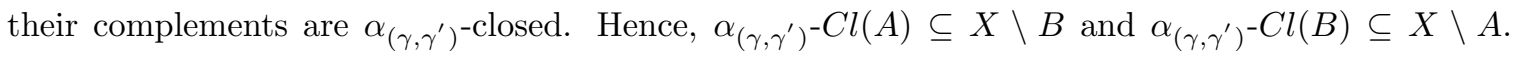
Therefore, $A$ and $B$ are $\alpha_{\left(\gamma, \gamma^{\prime}\right)}$-separated.

(3) If $A$ and $B$ are $\alpha_{\left(\gamma, \gamma^{\prime}\right)}$-open, then $X \backslash A$ and $X \backslash B$ are $\alpha_{\left(\gamma, \gamma^{\prime}\right)}$-closed. Since $H \subseteq X \backslash B, \alpha_{\left(\gamma, \gamma^{\prime}\right)}{ }^{-}$ $C l(H) \subseteq \alpha_{\left(\gamma, \gamma^{\prime}\right)} C l(X \backslash B)=X \backslash B$ and so $\alpha_{\left(\gamma, \gamma^{\prime}\right)^{-}} C l(H) \cap B=\phi$. Thus $G \cap \alpha_{\left(\gamma, \gamma^{\prime}\right)^{-}} C l(H)=\phi$. Similarly, $H \cap \alpha_{\left(\gamma, \gamma^{\prime}\right)} C l(G)=\phi$. Hence $H$ and $G$ are $\alpha_{\left(\gamma, \gamma^{\prime}\right)}$-separated. If $A$ and $B$ are $\alpha_{\left(\gamma, \gamma^{\prime}\right)}$-closed, then $\alpha_{\left(\gamma, \gamma^{\prime}\right)^{-}} C l(H) \subseteq A$ and $\alpha_{\left(\gamma, \gamma^{\prime}\right)}-C l(G) \subseteq B$. Thus, $H$ and $G$ are $\alpha_{\left(\gamma, \gamma^{\prime}\right)^{-}}$separated.

Theorem 3.2. The sets $A$ and $B$ of a space $X$ are $\alpha_{\left(\gamma, \gamma^{\prime}\right)}$-separated if and only if there exist $U$ and $V$ in $\alpha O(X, \tau)_{\left(\gamma, \gamma^{\prime}\right)}$ such that $A \subseteq U, B \subseteq V$ and $A \cap V=\phi$ and $B \cap U=\phi$.

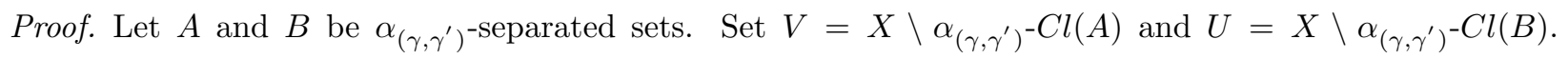
Then $U, V \in \alpha O(X, \tau)_{\left(\gamma, \gamma^{\prime}\right)}$ such that $A \subseteq U, B \subseteq V$ and $A \cap V=\phi, B \cap U=\phi$. On the other hand, let $U, V \in \alpha O(X, \tau)_{\left(\gamma, \gamma^{\prime}\right)}$ such that $A \subseteq U, B \subseteq V$ and $A \cap V=\phi, B \cap U=\phi$. Since $X \backslash V$ and $X \backslash U$ are $\alpha_{\left(\gamma, \gamma^{\prime}\right)^{-}}$ closed, then $\alpha_{\left(\gamma, \gamma^{\prime}\right)^{-}} C l(A) \subseteq X \backslash V \subseteq X \backslash B$ and $\alpha_{\left(\gamma, \gamma^{\prime}\right)^{-}} C l(B) \subseteq X \backslash U \subseteq X \backslash A$. Thus $\alpha_{\left(\gamma, \gamma^{\prime}\right)^{-}} C l(A) \cap B=\phi$ and $\alpha_{\left(\gamma, \gamma^{\prime}\right)}-C l(B) \cap A=\phi$.

Theorem 3.3. In any topological space $(X, \tau)$, the following statements are equivalent:

(1) $\phi$ and $X$ are the only $\alpha_{\left(\gamma, \gamma^{\prime}\right)}$-open and $\alpha_{\left(\gamma, \gamma^{\prime}\right)}$-closed sets in $X$.

(2) $X$ is not the union of two disjoint nonempty $\alpha_{\left(\gamma, \gamma^{\prime}\right)}$-open sets.

(3) $X$ is not the union of two disjoint nonempty $\alpha_{\left(\gamma, \gamma^{\prime}\right)^{-}}$-closed sets.

(4) $X$ is not the union of two nonempty $\alpha_{\left(\gamma, \gamma^{\prime}\right)}$-separated sets.

Proof. (1) $\Rightarrow(2)$ : Suppose (2) is false and that $X=A \cup B$, where $A, B$ are disjoint nonempty $\alpha_{\left(\gamma, \gamma^{\prime}\right)}$-open sets. Since $X \backslash A=B$ is $\alpha_{\left(\gamma, \gamma^{\prime}\right)}{ }^{\text {-open }}$ and nonempty, we have that $A$ is a nonempty proper $\alpha_{\left(\gamma, \gamma^{\prime}\right)}$-open and $\alpha_{\left(\gamma, \gamma^{\prime}\right)}$-closed set in $X$, which shows that (1) is false.

$(2) \Leftrightarrow(3)$ : This is clear. 


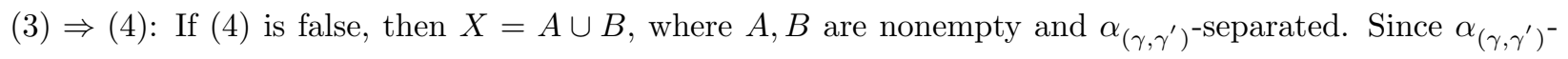
$C l(B) \cap A=\phi$, we conclude that $\alpha_{\left(\gamma, \gamma^{\prime}\right)} C l(B) \subseteq B$, so $B$ is $\alpha_{\left(\gamma, \gamma^{\prime}\right)^{-}}$-closed. Similarly, $A$ must be $\alpha_{\left(\gamma, \gamma^{\prime}\right)}$-closed. Therefore, (3) is false.

$(4) \Rightarrow(1)$ : Suppose (1) is false and that $A$ is a nonempty proper $\alpha_{\left(\gamma, \gamma^{\prime}\right)}$-open and $\alpha_{\left(\gamma, \gamma^{\prime}\right)}$-closed subset of $X$. Then, $B=X \backslash A$ is nonempty, $\alpha_{\left(\gamma, \gamma^{\prime}\right)}$-open and $\alpha_{\left(\gamma, \gamma^{\prime}\right)}$-closed, so $A$ and $B$ are $\alpha_{\left(\gamma, \gamma^{\prime}\right)^{-s e p a r a t e d}}$ and $X=A \cup B$, so (4) is false.

Definition 3.2. A subset $C$ of a space $X$ is said to be $\alpha_{\left(\gamma, \gamma^{\prime}\right)}$-disconnected if there are nonempty $\alpha_{\left(\gamma, \gamma^{\prime}\right)^{-}}$ separated subsets $A$ and $B$ of $X$ such that $C=A \cup B$, otherwise $C$ is called $\alpha_{\left(\gamma, \gamma^{\prime}\right)}$-connected. If $C$ is $\alpha_{\left(\gamma, \gamma^{\prime}\right)}$-disconnected, such a pair of sets $A, B$ will be called an $\alpha_{\left(\gamma, \gamma^{\prime}\right)}$-disconnection of $C$.

Example 3.3. Let $X=\{1,2,3\}$ and $\tau=\{\phi, X,\{1\},\{2\},\{1,2\},\{2,3\}\}$. For each $A \in \alpha O(X)$, we define two operations $\gamma$ and $\gamma^{\prime}$, respectively, by

$$
A^{\gamma}=A^{\gamma^{\prime}}= \begin{cases}A & \text { if } 3 \in A \\ C l(A) & \text { if } 3 \notin A .\end{cases}
$$

Then, $X$ is $\alpha_{\left(\gamma, \gamma^{\prime}\right)}$-disconnected because there exist a pair $\{1\},\{2,3\}$ subsets of $X$ such that $\{1\} \cup\{2,3\}=X$, and $\left(\alpha_{\left(\gamma, \gamma^{\prime}\right)}-C l(\{1\}) \cap\{2,3\}\right) \cup\left(\{1\} \cap \alpha_{\left(\gamma, \gamma^{\prime}\right)}-C l(\{2,3\})\right)=(\{1\} \cap\{2,3\}) \cup(\{1\} \cap\{2,3\})=\phi$.

Example 3.4. Let $X=\{1,2,3\}$ and $\tau=\{\phi, X,\{1\},\{3\},\{1,3\}\}$. For each $A \in \alpha O(X)$, we define two operations $\gamma$ and $\gamma^{\prime}$, respectively, by

$$
A^{\gamma}=A^{\gamma^{\prime}}= \begin{cases}A & \text { if } 2 \in A \\ X & \text { if } 2 \notin A .\end{cases}
$$

Then, $X$ is $\alpha_{\left(\gamma, \gamma^{\prime}\right)}$-connected, since there does not exist a pair $A, B$ of nonempty $\alpha_{\left(\gamma, \gamma^{\prime}\right)^{-}}$-separated subsets of $X$ such that $X=A \cup B$.

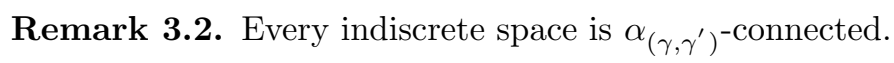

Remark 3.3. Every discrete space contains more than one element is $\alpha_{\left(i d, i d^{\prime}\right)}$-disconnected.

Remark 3.4. A space $X$ is $\alpha_{\left(\gamma, \gamma^{\prime}\right)}$-connected if any (therefore all) of the conditions (1) - (4) in Theorem 3.3 hold.

Remark 3.5. According to the Definition 3.2 and Remark 3.4, a space $X$ is $\alpha_{\left(\gamma, \gamma^{\prime}\right)}$-disconnected if we can write $X=A \cup B$, where the following (equivalent) statements are true:

(1) $A$ and $B$ are disjoint, nonempty and $\alpha_{\left(\gamma, \gamma^{\prime}\right)}$-open.

(2) $A$ and $B$ are disjoint, nonempty and $\alpha_{\left(\gamma, \gamma^{\prime}\right)}$-closed.

(3) $A$ and $B$ are nonempty and $\alpha_{\left(\gamma, \gamma^{\prime}\right)}$-separated. 
Theorem 3.4. A space $X$ is $\alpha_{\left(\gamma, \gamma^{\prime}\right)}$-disconnected if and only if there exists a nonempty proper subset A of $X$ which is both $\alpha_{\left(\gamma, \gamma^{\prime}\right)}$-open and $\alpha_{\left(\gamma, \gamma^{\prime}\right)}$-closed in $X$.

Proof. Follows from Remark 3.5.

Definition 3.3. Let $A$ be a subset of a space $X$, then the $\alpha_{\left(\gamma, \gamma^{\prime}\right)^{-}}$-boundary of $A$ is defined as $\alpha_{\left(\gamma, \gamma^{\prime}\right)^{-}}$ $C l(A) \backslash \alpha_{\left(\gamma, \gamma^{\prime}\right)}-\operatorname{Int}(A)$ and is denoted by $\alpha_{\left(\gamma, \gamma^{\prime}\right)}-B d(A)$.

Proposition 3.1. Let $A$ be any subset of a topological space $(X, \tau)$. Then, the following statements are hold:

(1) $\alpha_{\left(\gamma, \gamma^{\prime}\right)}-C l(A)=\alpha_{\left(\gamma, \gamma^{\prime}\right)}-\operatorname{Int}(A) \cup \alpha_{\left(\gamma, \gamma^{\prime}\right)}-B d(A)$.

(2) $\alpha_{\left(\gamma, \gamma^{\prime}\right)^{-}} B d(A)=\alpha_{\left(\gamma, \gamma^{\prime}\right)^{-}} C l(A) \cap \alpha_{\left(\gamma, \gamma^{\prime}\right)^{-}} C l(X \backslash A)$.

Proof. Obvious.

Theorem 3.5. A space $X$ is $\alpha_{\left(\gamma, \gamma^{\prime}\right)}$-connected if and only if every nonempty proper subset of $X$ has a nonempty $\alpha_{\left(\gamma, \gamma^{\prime}\right)}$-boundary.

Proof. Suppose that a nonempty proper subset $A$ of an $\alpha_{\left(\gamma, \gamma^{\prime}\right)^{-}}$-connected space $X$ has empty $\alpha_{\left(\gamma, \gamma^{\prime}\right)^{-}}$ boundary. Since $\alpha_{\left(\gamma, \gamma^{\prime}\right)} C l(A)=\alpha_{\left(\gamma, \gamma^{\prime}\right)^{-}} \operatorname{Int}(A) \cup \alpha_{\left(\gamma, \gamma^{\prime}\right)^{-}} B d(A)$. Thus, $A$ is both $\alpha_{\left(\gamma, \gamma^{\prime}\right)^{-}}$-closed and $\alpha_{\left(\gamma, \gamma^{\prime}\right)^{-}}$ open. By Theorem 3.4, $X$ is $\alpha_{\left(\gamma, \gamma^{\prime}\right)}$-disconnected. This contradiction, hence proves that $A$ has a nonempty $\alpha_{\left(\gamma, \gamma^{\prime}\right)}$-boundary.

Conversely, suppose $X$ is $\alpha_{\left(\gamma, \gamma^{\prime}\right)}$-disconnected. Then by Theorem 3.4, $X$ has a nonempty proper subset $A$ which is both $\alpha_{\left(\gamma, \gamma^{\prime}\right)^{-}}$-closed and $\alpha_{\left(\gamma, \gamma^{\prime}\right)^{-o p e n}}$. Then, $\alpha_{\left(\gamma, \gamma^{\prime}\right)}-C l(A)=A, \alpha_{\left(\gamma, \gamma^{\prime}\right)}-C l(X \backslash A)=X \backslash A$ and

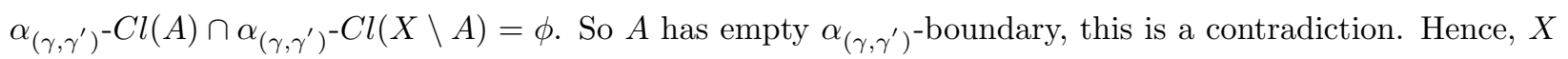

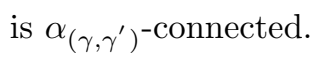

Lemma 3.1. Suppose $M, N$ are $\alpha_{\left(\gamma, \gamma^{\prime}\right)}$-separated subsets of $X$. If $C \subseteq M \cup N$ and $C$ is $\alpha_{\left(\gamma, \gamma^{\prime}\right)}$-connected, then $C \subseteq M$ or $C \subseteq N$.

Proof. Since $C \cap M \subseteq M$ and $C \cap N \subseteq N$, then $C \cap M$ and $C \cap N$ are $\alpha_{\left(\gamma, \gamma^{\prime}\right)}$-separated and $C=C \cap(M \cup N)=$ $(C \cap M) \cup(C \cap N)$. But $C$ is $\alpha_{\left(\gamma, \gamma^{\prime}\right)}$-connected so $(C \cap M)$ and $(C \cap N)$ can not form an $\alpha_{\left(\gamma, \gamma^{\prime}\right)}$-disconnection of $C$. Therefore, either $C \cap M=\phi$, so $C \subseteq N$ or $C \cap N=\phi$, so $C \subseteq M$.

Theorem 3.6. Suppose $C$ and $C_{i}(i \in I)$ are $\alpha_{\left(\gamma, \gamma^{\prime}\right)}$-connected subsets of $X$ and that for each $i, C_{i}$ and $C$ are not $\alpha_{\left(\gamma, \gamma^{\prime}\right)}$-separated. Then, $S=C \cup C_{i}$ is $\alpha_{\left(\gamma, \gamma^{\prime}\right)}$-connected.

Proof. Suppose that $S=M \cup N$, where $M$ and $N$ are $\alpha_{\left(\gamma, \gamma^{\prime}\right)}$-separated. By Lemma 3.1, either $C \subseteq M$ or $C \subseteq N$. Without loss of generality, assume $C \subseteq M$. By the same reasoning we conclude that for each $i$, 
either $C_{i} \subseteq M$ or $C_{i} \subseteq N$. But if some $C_{i} \subseteq N$, then $C$ and $C_{i}$ would be $\alpha_{\left(\gamma, \gamma^{\prime}\right)}$-separated. Hence every $C_{i} \subseteq M$. Therefore, $N=\phi$ and the pair $M, N$ is not an $\alpha_{\left(\gamma, \gamma^{\prime}\right)}$-disconnection of $S$.

Corollary 3.1. Suppose that for each $i \in I, C_{i}$ is an $\alpha_{\left(\gamma, \gamma^{\prime}\right)}$-connected subset of $X$ and that for all $i \neq j$, $C_{i} \cap C_{j} \neq \phi$. Then, $\cup\left\{C_{i}: i \in I\right\}$ is $\alpha_{\left(\gamma, \gamma^{\prime}\right)}$-connected.

Proof. If $I=\phi$, then $\cup\left\{C_{i}: i \in I\right\}=\phi$ is $\alpha_{\left(\gamma, \gamma^{\prime}\right)}$-connected. If $I \neq \phi$, pick $i_{0} \in I$ and let $C_{i_{0}}$ be the central set $C$ in Theorem 3.6. For all $i \in I, C_{i} \cap C_{i_{0}} \neq \phi$, so $C_{i}$ and $C_{i_{0}}$ are not $\alpha_{\left(\gamma, \gamma^{\prime}\right)}$-separated. By Theorem 3.6, $\cup\left\{C_{i}: i \in I\right\}$ is $\alpha_{\left(\gamma, \gamma^{\prime}\right)}$-connected.

Corollary 3.2. Suppose that for all $x, y \in X$, there exists an $\alpha_{\left(\gamma, \gamma^{\prime}\right)}$-connected set $C_{x y} \subseteq X$ with $x, y \in C_{x y}$. Then, $X$ is $\alpha_{\left(\gamma, \gamma^{\prime}\right)}$-connected.

Proof. Certainly $X=\phi$ is $\alpha_{\left(\gamma, \gamma^{\prime}\right)}$-connected. If $X \neq \phi$, choose $a \in X$. By hypothesis there is, for each $y \in X$, an $\alpha_{\left(\gamma, \gamma^{\prime}\right)}$-connected set $C_{a y}$ containing both $a$ and $y$. By Corollary 3.1, $X=\cup\left\{C_{a y}: y \in X\right\}$ is $\alpha_{\left(\gamma, \gamma^{\prime}\right)}$-connected.

Corollary 3.3. Suppose $C$ is an $\alpha_{\left(\gamma, \gamma^{\prime}\right)}$-connected subset of $X$ and $A \subseteq X$. If $C \subseteq A \subseteq \alpha_{\left(\gamma, \gamma^{\prime}\right)}{ }^{-C l(C) \text {, then }}$ $A$ is $\alpha_{\left(\gamma, \gamma^{\prime}\right)}$-connected.

Proof. For each $a \in A,\{a\}$ and $C$ are not $\alpha_{\left(\gamma, \gamma^{\prime}\right)}$-separated. By Theorem 3.6, $A=C \cup \bigcup\{\{a\}: a \in A\}$ is $\alpha_{\left(\gamma, \gamma^{\prime}\right)}$-connected.

Remark 3.6. In particular, the $\alpha_{\left(\gamma, \gamma^{\prime}\right)}$-closure of an $\alpha_{\left(\gamma, \gamma^{\prime}\right)}$-connected set is $\alpha_{\left(\gamma, \gamma^{\prime}\right)}$-connected.

Theorem 3.7. Let $f:(X, \tau) \rightarrow(Y, \sigma)$ be a function. Consider the following statements.

(1) $f$ is $\left(\alpha_{\left(\gamma, \gamma^{\prime}\right)}, \alpha_{\left(\beta, \beta^{\prime}\right)}\right)$-continuous.

(2) $f^{-1}(V) \subseteq \alpha_{\left(\gamma, \gamma^{\prime}\right)}-\operatorname{Int}\left(f^{-1}(V)\right)$ for every $\alpha_{\left(\beta, \beta^{\prime}\right)}$-open set $V$ of $Y$.

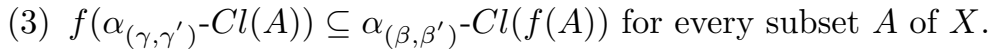

(4) $\alpha_{\left(\gamma, \gamma^{\prime}\right)}-C l\left(f^{-1}(B)\right) \subseteq f^{-1}\left(\alpha_{\left(\beta, \beta^{\prime}\right)}-C l(B)\right)$ for every subset $B$ of $Y$.

Then, the following implications are true: $(1) \Rightarrow(2) \Rightarrow(3) \Rightarrow(4)$.

Proof. $(1) \Rightarrow(2)$. Let $V$ be any $\alpha_{\left(\beta, \beta^{\prime}\right)}$-open set of $Y$ and $x \in f^{-1}(V)$. Then, $f(x) \in V$. Since $f$ is $\left(\alpha_{\left(\gamma, \gamma^{\prime}\right)}, \alpha_{\left(\beta, \beta^{\prime}\right)}\right)$-continuous, there exists an $\alpha_{\left(\gamma, \gamma^{\prime}\right)}$-open set $U$ of $X$ containing $x$ such that $f(U) \subseteq V$ and hence $U \subseteq f^{-1}(V)$, this implies that $x \in \alpha_{\left(\gamma, \gamma^{\prime}\right)^{-}} \operatorname{Int}\left(f^{-1}(V)\right)$. Thus, it follows that $f^{-1}(V) \subseteq \alpha_{\left(\gamma, \gamma^{\prime}\right)^{-}}$ $\operatorname{Int}\left(f^{-1}(V)\right)$.

$(2) \Rightarrow(3)$. Let $A$ be any subset of $X$ and $f(x) \notin \alpha_{\left(\beta, \beta^{\prime}\right)}-C l(f(A))$. Then, by Proposition 2.2 , there exists an $\alpha_{\left(\beta, \beta^{\prime}\right)}$-open set $V$ of $Y$ containing $f(x)$ such that $V \cap f(A)=\phi$ and hence $f^{-1}(V) \cap A=\phi$. Also $f(x) \in V$ implies $x \in f^{-1}(V)$. Then by (2) we obtain that $x \in \alpha_{\left(\gamma, \gamma^{\prime}\right)} \operatorname{Int}\left(f^{-1}(V)\right)$. Hence, there exists an 
$\alpha_{\left(\gamma, \gamma^{\prime}\right)^{-}}$open set $U$ of $X$ containing $x$ such that $U \subseteq f^{-1}(V)$. Then $U \cap A=\phi$ and so $x \notin \alpha_{\left(\gamma, \gamma^{\prime}\right)}-C l(A)$. This implies $f(x) \notin f\left(\alpha_{\left(\gamma, \gamma^{\prime}\right)^{-}} C l(A)\right)$. Thus, $f\left(\alpha_{\left(\gamma, \gamma^{\prime}\right)^{-}} C l(A)\right) \subseteq \alpha_{\left(\beta, \beta^{\prime}\right)}-C l(f(A))$.

$(3) \Rightarrow(4)$. Let $B$ be any subset of $Y$. Since $f\left(f^{-1}(B)\right) \subseteq B$, so, we have $\alpha_{\left(\beta, \beta^{\prime}\right)}-C l\left(f\left(f^{-1}(B)\right)\right) \subseteq \alpha_{\left(\beta, \beta^{\prime}\right)^{-}}$ $C l(B)$. Also, $f^{-1}(B) \subseteq X$, then by (3), we have $f\left(\alpha_{\left(\gamma, \gamma^{\prime}\right)^{-}} C l\left(f^{-1}(B)\right)\right) \subseteq \alpha_{\left(\beta, \beta^{\prime}\right)^{-}} C l\left(f\left(f^{-1}(B)\right)\right) \subseteq \alpha_{\left(\beta, \beta^{\prime}\right)^{-}}$ $C l(B)$. Thus, $\alpha_{\left(\gamma, \gamma^{\prime}\right)}-C l\left(f^{-1}(B)\right) \subseteq f^{-1}\left(\alpha_{\left(\beta, \beta^{\prime}\right)}-C l(B)\right)$.

Corollary 3.4. Let $f: X \rightarrow Y$ be an $\left(\alpha_{\left(\gamma, \gamma^{\prime}\right)}, \alpha_{\left(\beta, \beta^{\prime}\right)}\right)$-continuous and injective function. If $K$ is $\alpha_{\left(\gamma, \gamma^{\prime}\right)^{-}}$ connected in $X$, then $f(K)$ is $\alpha_{\left(\beta, \beta^{\prime}\right)}$-connected in Y.

Proof. Suppose that $f(K)$ is $\alpha_{\left(\beta, \beta^{\prime}\right)}$-disconnected in $Y$. There exist two $\alpha_{\left(\beta, \beta^{\prime}\right)}$-separated sets $P$ and $Q$ of $Y$ such that $f(K)=P \cup Q$. Set $A=K \cap f^{-1}(P)$ and $B=K \cap f^{-1}(Q)$. Since $f(K) \cap P \neq \phi$, then $K \cap f^{-1}(P) \neq \phi$ and so $A \neq \phi$. Similarly $B \neq \phi$. Now, $A \cup B=\left(K \cap f^{-1}(P)\right) \cup\left(K \cap f^{-1}(Q)\right)=K \cap\left(f^{-1}(P) \cup f^{-1}(Q)\right)=$ $K \cap f^{-1}(P \cup Q)=K \cap f^{-1}(f(K))=K$. Since $f$ is $\left(\alpha_{\left(\gamma, \gamma^{\prime}\right)}, \alpha_{\left(\beta, \beta^{\prime}\right)}\right)$-continuous, then by Theorem 3.7 , $\alpha_{\left(\gamma, \gamma^{\prime}\right)^{-}} C l\left(f^{-1}(Q)\right) \subseteq f^{-1}\left(\alpha_{\left(\beta, \beta^{\prime}\right)}-C l(Q)\right)$ and $B \subseteq f^{-1}(Q)$, then $\alpha_{\left(\gamma, \gamma^{\prime}\right)} C l(B) \subseteq f^{-1}\left(\alpha_{\left(\beta, \beta^{\prime}\right)}-C l(Q)\right)$. Since $P \cap \alpha_{\left(\beta, \beta^{\prime}\right)^{-}} C l(Q)=\phi$, then $A \cap \alpha_{\left(\gamma, \gamma^{\prime}\right)^{-}} C l(B) \subseteq A \cap f^{-1}\left(\alpha_{\left(\beta, \beta^{\prime}\right)}-C l(Q)\right) \subseteq f^{-1}(P) \cap f^{-1}\left(\alpha_{\left(\beta, \beta^{\prime}\right)^{-}} C l(Q)\right)=\phi$ and then $A \cap \alpha_{\left(\gamma, \gamma^{\prime}\right)}-C l(B)=\phi$. Similarly, $B \cap \alpha_{\left(\gamma, \gamma^{\prime}\right)^{-}} C l(A)=\phi$. Thus, $A$ and $B$ are $\alpha_{\left(\gamma, \gamma^{\prime}\right)^{-s e p a r a t e d}}$ Therefore, $K$ is $\alpha_{\left(\gamma, \gamma^{\prime}\right)}$-disconnected, this is contradiction. Hence, $f(K)$ is $\alpha_{\left(\beta, \beta^{\prime}\right)}$-connected.

Theorem 3.8. If $f:(X, \tau) \rightarrow(Y, \sigma)$ is an onto $\left(\alpha_{\left(\gamma, \gamma^{\prime}\right)}, \alpha_{\left(\beta, \beta^{\prime}\right)}\right)$-continuous function and $X$ is $\alpha_{\left(\gamma, \gamma^{\prime}\right)^{-}}$ connected, then $Y$ is $\alpha_{\left(\beta, \beta^{\prime}\right)}$-connected.

Proof. Suppose that $Y$ is $\alpha_{\left(\beta, \beta^{\prime}\right)}$-disconnected and $A, B$ is an $\alpha_{\left(\beta, \beta^{\prime}\right)}$-disconnection of $Y$. By Remark 3.5, $A$ and $B$ are both $\alpha_{\left(\beta, \beta^{\prime}\right)}$-open sets. Since $f$ is $\left(\alpha_{\left(\gamma, \gamma^{\prime}\right)}, \alpha_{\left(\beta, \beta^{\prime}\right)}\right)$-continuous, so by Theorem $2.1, f^{-1}(A)$ and $f^{-1}(B)$ are both nonempty $\alpha_{\left(\gamma, \gamma^{\prime}\right)}$-open sets in $X$. Now, $f^{-1}(A) \cup f^{-1}(B)=f^{-1}(A \cup B)=f^{-1}(Y)=X$, and $f^{-1}(A) \cap f^{-1}(B)=f^{-1}(A \cap B)=f^{-1}(\phi)=\phi$. Then by Remark 3.5, $f^{-1}(A), f^{-1}(B)$ is a pair of $\alpha_{\left(\gamma, \gamma^{\prime}\right)}$-disconnection of $X$. This contradiction shows that $Y$ is $\alpha_{\left(\beta, \beta^{\prime}\right)}$-connected.

Corollary 3.5. For a bijective $\left(\alpha_{\left(\gamma, \gamma^{\prime}\right)}, \alpha_{\left(\beta, \beta^{\prime}\right)}\right)$-closed function $f: X \rightarrow Y$, if $C$ is $\alpha_{\left(\beta, \beta^{\prime}\right)}$-connected in $Y$, then $f^{-1}(C)$ is $\alpha_{\left(\gamma, \gamma^{\prime}\right)}$-connected in $X$.

Proof. Suppose that $f^{-1}(C)$ is $\alpha_{\left(\gamma, \gamma^{\prime}\right)}$-disconnected in $X$. There exist two $\alpha_{\left(\gamma, \gamma^{\prime}\right)}$-separated sets $M$ and $N$ of $X$ such that $f^{-1}(C)=M \cup N$. Set $K=C \cap f(M)$ and $L=C \cap f(N)$. Since $C=f(M) \cup f(N)$, then $C \cap f(M) \neq \phi$ and so $K \neq \phi$. Similarly $L \neq \phi$. Now, $K \cup L=(C \cap f(M)) \cup(C \cap f(N))=$ $C \cap(f(M) \cup f(N))=C \cap f(M \cup N)=C \cap f\left(f^{-1}(C)\right)=C$. Since $f$ is $\left(\alpha_{\left(\gamma, \gamma^{\prime}\right)}, \alpha_{\left(\beta, \beta^{\prime}\right)}\right)$-closed, then by Proposition 2.3, $\alpha_{\left(\beta, \beta^{\prime}\right)^{-}} C l(f(N)) \subseteq f\left(\alpha_{\left(\gamma, \gamma^{\prime}\right)^{-}} C l(N)\right)$ and $L \subseteq f(N)$, then $\alpha_{\left(\beta, \beta^{\prime}\right)^{-}} C l(L) \subseteq f\left(\alpha_{\left(\gamma, \gamma^{\prime}\right)^{-}} C l(N)\right)$. Since $M \cap \alpha_{\left(\gamma, \gamma^{\prime}\right)^{-}} C l(N)=\phi$, then $K \cap \alpha_{\left(\beta, \beta^{\prime}\right)^{-}} C l(L) \subseteq K \cap f\left(\alpha_{\left(\gamma, \gamma^{\prime}\right)^{-}} C l(N)\right) \subseteq f(M) \cap f\left(\alpha_{\left(\gamma, \gamma^{\prime}\right)^{-}} C l(N)\right)=\phi$ and then $K \cap \alpha_{\left(\beta, \beta^{\prime}\right)^{-}} C l(L)=\phi$. Similarly, $L \cap \alpha_{\left(\beta, \beta^{\prime}\right)} C l(K)=\phi$. Thus, $K$ and $L$ are $\alpha_{\left(\beta, \beta^{\prime}\right)^{-s e p a r a t e d}}$ Therefore, $C$ is $\alpha_{\left(\beta, \beta^{\prime}\right)}$-disconnected, this is contradiction. Hence, $f^{-1}(C)$ is $\alpha_{\left(\gamma, \gamma^{\prime}\right)}$-connected. 
Definition 3.4. A set $C$ is called a maximal $\alpha_{\left(\gamma, \gamma^{\prime}\right)}$-connected set if it is $\alpha_{\left(\gamma, \gamma^{\prime}\right)}$-connected and if $C \subseteq D \subseteq X$ where $D$ is $\alpha_{\left(\gamma, \gamma^{\prime}\right)^{-}}$-connected, then $C=D$. A maximal $\alpha_{\left(\gamma, \gamma^{\prime}\right)}$-connected subset $C$ of a space $X$ is called an

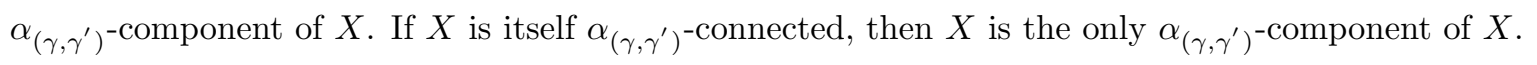

Theorem 3.9. For each $x \in X$, there is exactly one $\alpha_{\left(\gamma, \gamma^{\prime}\right)}$-component of $X$ containing $x$.

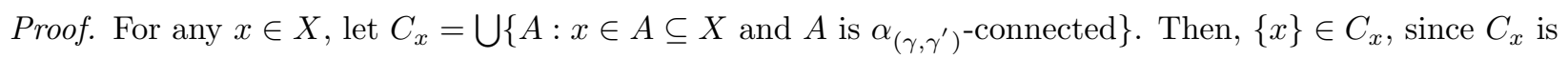
a union of $\alpha_{\left(\gamma, \gamma^{\prime}\right)}$-connected sets each containing $x, C_{x}$ is $\alpha_{\left(\gamma, \gamma^{\prime}\right)^{-}}$-connected by Corollary 3.1. If $C_{x} \subseteq D$ and $D$ is $\alpha_{\left(\gamma, \gamma^{\prime}\right)}$-connected, then $D$ was one of the sets $A$ in the collection whose union defines $C_{x}$, so $D \subseteq C_{x}$ and therefore $C_{x}=D$. Therefore, $C_{x}$ is an $\alpha_{\left(\gamma, \gamma^{\prime}\right)}$-component of $X$ that contains $x$.

Corollary 3.6. A space $X$ is the union of its $\alpha_{\left(\gamma, \gamma^{\prime}\right)}$-components.

Proof. Follows from Theorem 3.9.

Corollary 3.7. Two $\alpha_{\left(\gamma, \gamma^{\prime}\right)}$-components are either disjoint or coincide.

Proof. Let $C_{x}$ and $C_{y}$ be $\alpha_{\left(\gamma, \gamma^{\prime}\right)}$-components and $C_{x} \neq C_{y}$. If $p \in C_{x} \cap C_{y}$, then by Corollary 3.1, $C_{x} \cup C_{y}$ would be an $\alpha_{\left(\gamma, \gamma^{\prime}\right)}$-connected set strictly larger than $C_{x}$. Therefore, $C_{x} \cap C_{y}=\phi$.

Theorem 3.10. Each $\alpha_{\left(\gamma, \gamma^{\prime}\right)}$-connected subset of $X$ is contained in exactly one $\alpha_{\left(\gamma, \gamma^{\prime}\right)}$-component of $X$.

Proof. Let $A$ be an $\alpha_{\left(\gamma, \gamma^{\prime}\right)}$-connected subset of $X$ which is not in exactly one $\alpha_{\left(\gamma, \gamma^{\prime}\right)^{-}}$-component of $X$. Suppose

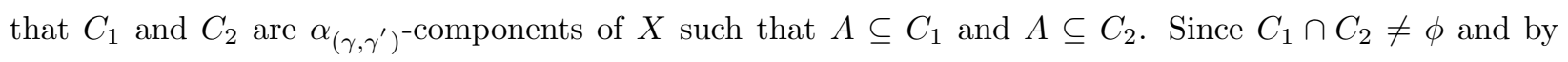
Corollary 3.1, $C_{1} \cup C_{2}$ is another $\alpha_{\left(\gamma, \gamma^{\prime}\right)}$-connected set which contains $C_{1}$ as well as $C_{2}$, a contradiction to the fact that $C_{1}$ and $C_{2}$ are $\alpha_{\left(\gamma, \gamma^{\prime}\right)}$-components. This proves that $A$ is contained in exactly one $\alpha_{\left(\gamma, \gamma^{\prime}\right)}$-component of $X$.

Theorem 3.11. A nonempty $\alpha_{\left(\gamma, \gamma^{\prime}\right)}$-connected subset of $X$ which is both $\alpha_{\left(\gamma, \gamma^{\prime}\right)}$-open and $\alpha_{\left(\gamma, \gamma^{\prime}\right)}$-closed is $\alpha_{\left(\gamma, \gamma^{\prime}\right)}$-component.

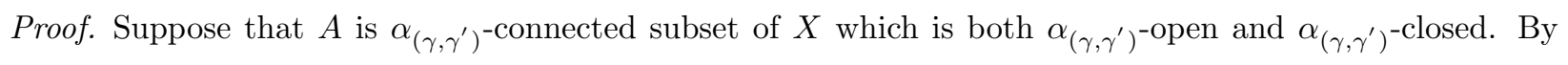
Theorem 3.10, $A$ is contained in exactly one $\alpha_{\left(\gamma, \gamma^{\prime}\right)}$-component $C$ of $X$. If $A$ is a proper subset of $C$, then $C=(C \cap A) \cup(C \cap(X \backslash A))$ and $(C \cap A),(C \cap(X \backslash A))$ is an $\alpha_{\left(\gamma, \gamma^{\prime}\right)}$-disconnection of $C$, which is a contradiction. Thus, $A=C$.

Theorem 3.12. Every $\alpha_{\left(\gamma, \gamma^{\prime}\right)}$-component of $X$ is $\alpha_{\left(\gamma, \gamma^{\prime}\right)}$-closed.

Proof. Suppose that $C$ is an $\alpha_{\left(\gamma, \gamma^{\prime}\right)}$-component of $X$. Then, by Remark 3.6, $\alpha_{\left(\gamma, \gamma^{\prime}\right)} C l(C)$ is $\alpha_{\left(\gamma, \gamma^{\prime}\right)}$-connected containing $\alpha_{\left(\gamma, \gamma^{\prime}\right)}$-component $C$ of $X$. This implies that $C=\alpha_{\left(\gamma, \gamma^{\prime}\right)} C l(C)$ and hence $C$ is $\alpha_{\left(\gamma, \gamma^{\prime}\right)}$-closed. 


\section{ReFERENCES}

[1] H. Z. Ibrahim, On a class of $\alpha_{\left(\gamma, \gamma^{\prime}\right)}$-open sets in a topological space, Acta Sci., Technol., 35 (3) (2013), 539-545.

[2] H. Z. Ibrahim, On $\alpha_{\left(\gamma, \gamma^{\prime}\right)}$-open sets in topological spaces, (submitted).

[3] H. Z. Ibrahim, On $\left(\alpha_{\left(\gamma, \gamma^{\prime}\right)}, \alpha_{\left(\beta, \beta^{\prime}\right)}\right)$-functions, (submitted).

[4] S. Mishra, On $\alpha$ - $\tau$-disconnectedness and $\alpha$ - $\tau$-connectedness in topological spaces, Acta Sci., Technol., 37 (3) (2015), 395 399.

[5] O. Njastad, On some classes of nearly open sets, Pac. J. Math. 15 (1965), 961-970. 